\title{
Scrotal Granular Cell Tumor: A Case Report
}

\author{
Brano Djenic ${ }^{1 *}$ and Kaveh Homayoon ${ }^{2}$ \\ ${ }^{1}$ General Surgery Resident, Department of Surgery, Maricopa Medical Center, USA
}

${ }^{2}$ Department of Surgery, Division of Urology, Maricopa Medical Center, USA

Submission: April 26, 2017; Published: May 05, 2017

*Corresponding author: Brano Djenic, Department of Surgery, Maricopa Medical Center, Phoenix, AZ 85008, USA, Tel: 2108630673;

Email: brano.djenic@mihs.org

\section{Introduction}

Previously referred to as granular cell myblastoma, granular cell neuroma, granular cell neurofibroma andgranular cell schwanomma, the Abrikossoff's tumor or today known as granular cell tumor (GCT), is classified as a neural lesion that is very distinct from neurofibromas, schwanommas or muscle tumors. Although they can occur in both males and females and at any age, they are more commonly seen in females (2:1) in fourth, fifth or sixth decade. Granular cell tumors occur more commonly in African Americans andthey are rare in children. Head and neck GCTs make up 75\%, with tongue being the most common location; reports of breast lesions, GI tact involvement, and miscellaneous skin and soft tissue GCTs have been reported. Usual presentation is a solitary, painless nodule in dermis or sub cutis, less than 3 centimeters in size and less than 6 months in duration. $10-15 \%$ of granular cell tumors occur at multiple sites, with reports of up to 50 nodules; familial incidence is uncommon. We are presenting this case as a rare entity which does not fit the average epidemiological criteria of granular cell tumors: young, African American male, with a painless scrotal mass present for a decade, likely with previous GCTs in the past.

\section{Methods}

This is a case report and a review of the literature. This case study was reviewed and approved by IRB.Diagnostic work up and follow up care were carried out at Maricopa Medical Center, in Phoenix, Arizona, USA.

\section{Case Presentation}

The patient is a 17 year old healthy boy who presented to the urology clinic with a painless, posterior scrotal mass, about $2 \mathrm{~cm}$ in size and not increasing, which has been present for over 10 years and has been worrisome. He had a similar lesion on his anterior neck prior to the current one, near the esophagus, which was excised at a different facility; patient and parents do not have records of previous operation or pathological report, however, they do report that they were told that lesion is benign. No recurrence has occurred at the neck site [1]. The patient wanted scrotal mass removed, as well. Excision of the scrotal mass was uneventful. Primary closure of the scrotum was successful. Post-operative visits revealed nicely healing incision without drainage, erythema or pain. Pathological reports revealed a benign granular cell tumor. Patient and family are aware of the benign nature of the lesion as well as its chance for recurrence. Follow up is on as needed basis.

\section{Discussion}

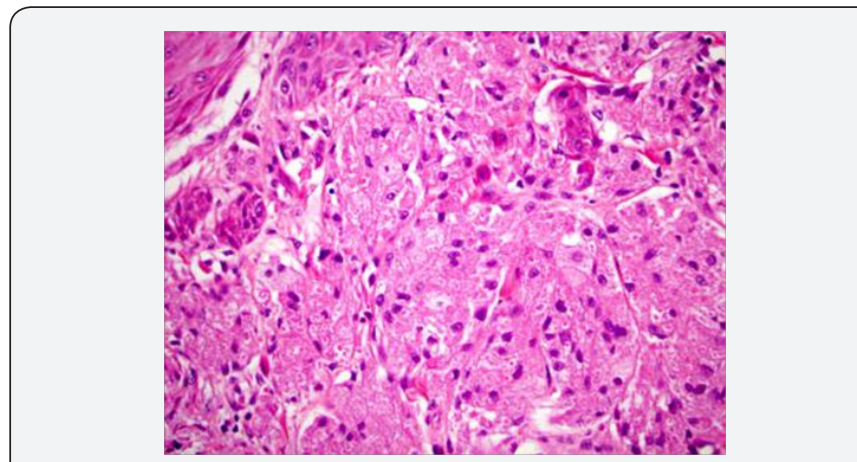

Figure 1: Granular cell tumor underneath the squamous epithelium.

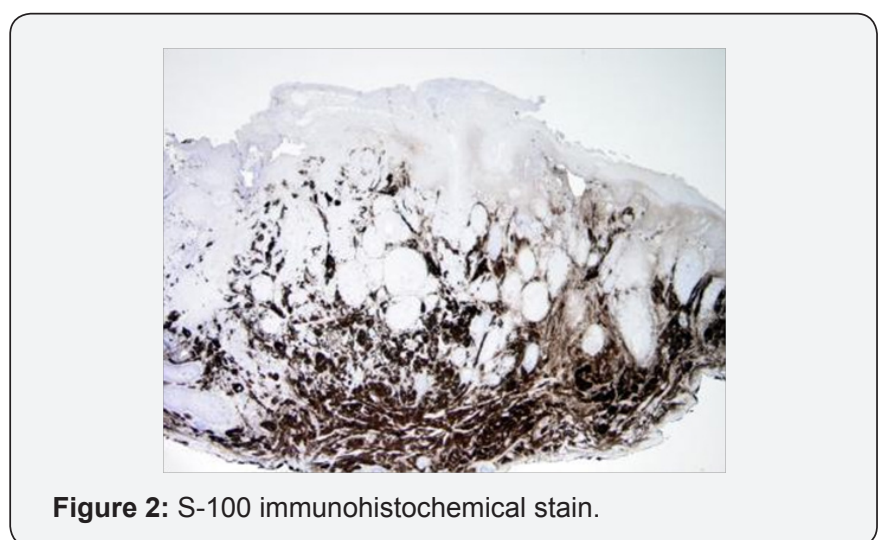

GCTs tend to be poorly circumscribed nodules, with pale yellow-tan cross sections. $67 \%$ or more are dermal, subcutaneous 
or submucosal (Figure 1). The cells of these tumors are rounded, polygonal, with nuclei ranging from small and dark to large with chromatin. Mild to moderate amounts of nuclear atypia are often seen but are not diagnostic of malignancy. The granules, which are phagolysosomes, are PAS-positive, diastase-resistant. These tumors are immunoreactice for S-100 protein (Figure 2), neuron-specific enolase, laminin and various myelin proteins; they are also positive for panmacrophage antigen CD68 and negative for neurofilament proteins, glial fibillary acidic protein (GFAP) and smooth muscle actin (SMA). Differential diagnoses which should be considered when examining granular tumors include rhabdomyoma, hibernoma, fibroxanthoma as well as squamous cell carcinoma (Figure 3). 98\% of these tumors are benign and surgical excision is curative. However, in those that are malignant, the course and presentation differ: they are encountered later in life, they are larger in size and they are present for longer periods of time when compared to benign GCTs. Recurrence commonlyoccurs one year after removal and typically precedes metastasis, which occurs through lymphatics and blood stream and has been reported in lung, liver and bone most commonly. Careful pathological examination and complete surgical excision are necessary to ensure proper diagnosis and best chance of cure.

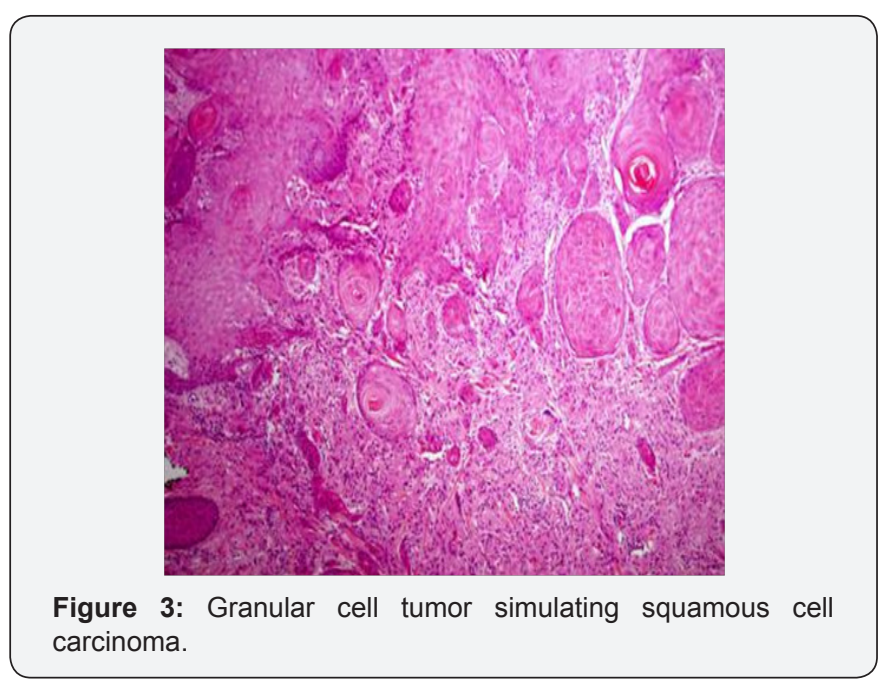

\section{References}

1. Peterson LJ (1974) Granular-cell tumor. Review of the literature and report of a case. Oral Surg Oral Med Oral Pathol 37(5): 728-735.

2. Rejas RA, Campos MS, Cortes AR, Pinto DD, de Sousa SC (2011) The neural histogenetic origin of the oral granular cell tumor: an immunohistochemical evidence. Med Oral Patol Oral Cir Bucal 16(1): e6-10.

3. http://emedicine.medscape.com/article/282430-differential

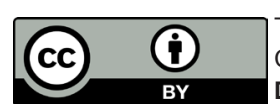

This work is licensed under Creative Commons Attribution 4.0 Licens DOI: 10.19080/OAJS.2017.04.555634 